\title{
The Correlation between the Orbital Volume Ratio and Enophthalmos in Unoperated Blowout Fractures
}

\author{
Su Hyun Choi, Dong Hee Kang, Ja Hea Gu \\ Department of Plastic and Reconstructive Surgery, Dankook University Hospital, Dankook University College of Medicine, Cheonan, Korea
}

Background Enophthalmos may not appear immediately after trauma due to periorbital swelling in a blowout fracture, and preoperative measurements of enophthalmos cannot be used as a reliable guideline. It is important to predict the eventual final extent of enophthalmos in order to determine whether to perform surgery, and there have been several attempts to predict the degree of late enophthalmos using preoperative orbital volume. The purpose of this study is to investigate the correlation between the orbital volume ratio (OVR) with final enophthalmos and the palpebral fissure, and to find the OVR that induced $2 \mathrm{~mm}$ of enophthalmos in unilateral unoperated blowout fractures.

Methods We retrospectively reviewed the medical records of 38 patients and divided them into 3 groups, determined by the fracture location. The relationships between the OVR and both the degree of enophthalmos and the palpebral fissure ratio (PFR) were assessed and, in particular, the OVR that induced $2 \mathrm{~mm}$ of enophthalmos was sought.

Results Enophthalmos increased in proportion to the OVR, and there was a highly significant correlation between the increase in the OVR and the degree of enophthalmos $(P<0.05)$. On the other hand, there was no correlation between OVR and PFR $(P>0.05)$. The OVR that induced 2-mm enophthalmos was $112.18 \%$.

Conclusions The final degree of enophthalmos can be estimated by the preoperative measurement of OVR. Preoperative measurements of OVR can be used as quantitative values to predict the final degree of enophthalmos in pure blowout fractures.

Keywords Orbital fractures / Enophthalmos / Orbit
Correspondence: Dong Hee Kang Department of Plastic and Reconstructive Surgery, Dankook University Hospital, Dankook University College of Medicine, 201 Manghyang-ro, Dongnam-gu, Cheonan 31116, Korea Tel: +82-41-550-6477 Fax: +82-41-554-6477 E-mail:dhkcool@daum.net

This article was presented at the 72 nd Congress of the Korean Society of Plastic and Reconstructive Surgeons, on November 7, 2014 in Seoul, Republic of Korea.

No potential conflict of interest relevant to this article was reported.

Received: 12 Apr $2016 \bullet$ Revised: 15 Oct $2016 \bullet$ Accepted: 18 Oct 2016

pISSN: 2234-6163 • elSSN: 2234-6171 • https://doi.org/10.5999/aps.2016.43.6.518• Arch Plast Surg 2016;43:518-522

\section{INTRODUCTION}

The indications for open reduction of orbital fractures have traditionally been considered to be the presence of symptoms such as limitation of extraocular muscle motion, radiologic evidence of extensive fracture (fracture size $>2 \mathrm{~cm}^{2}$ ), and enophthalmos produced by an orbital volume (OV) expansion [1]. Among these indications, enophthalmos may not appear immediately after trauma due to periorbital swelling; furthermore, it is difficult to detect enophthalmos before surgery because of residual swelling $[2,3]$. It has long been known that the cause of enophthalmos is a discrepancy between the volume of orbital soft tissue and the size of the bony orbital cavity; in addition, it is understood that the degree of enophthalmos increases proportion- 
ally with the increase in the OV [3]. However, the periorbital swelling caused by trauma makes it difficult to measure the exact amount of enophthalmos, and measurements of the final stable degree of enophthalmos are frequently delayed by several months until after the resolution of periorbital swelling [2]. Therefore, preoperative measurements cannot be used as a reliable guideline for the orbital surgeon, and it is important to predict the eventual final extent of enophthalmos in order to determine the need for surgery in orbital fracture patients. There have been several attempts to predict the extent of late enophthalmos using preoperative OV measurements with orbital computed tomography $(\mathrm{CT})[4,5]$. However, volumetric measurements of the orbit have not previously been used for surgical guidelines.

In this study, we measured the orbital volume ratio (OVR) using facial bone $\mathrm{CT}$ and examined its relationship with the final extent of enophthalmos and palpebral fissure in unoperated unilateral blowout fracture patients. Through our study, we investigated the correlation between the final enophthalmos and the OVR, and sought to determine the reliability of OVR measurements that could estimate the eventual stable degree of enophthalmos, as well as to find the OVR that induced $2 \mathrm{~mm}$ of enophthalmos; many studies have considered this the cosmetically significant threshold in blowout fractures [6-8].

\section{METHODS}

\section{Subjects}

We retrospectively reviewed the medical records of 38 unoperated patients who had experienced unilateral blowout fracture between April 2010 and March 2016. Thirty-eight patients satisfied the following criteria: (1) old unilateral pure blowout fracture with at least a 6-month follow-up; (2) no history of medical diseases that might have altered the OV or palpebral height; and (3) age < 65 years, to exclude the influence of senile change on palpebral fissure height. The patients were divided into 3 groups, depending on the anatomic location of the fracture based on a facial CT scan: group I (inferior wall, 7 patients), group M (medial wall, 19 patients), and group IM (inferomedial wall, 12 patients).

\section{Facial CT scans and OVR measurements}

Three-dimensional CT images (GE Lightspeed VCT; GE Medical System, Milwaukee, WI, USA) taken within 1 week after trauma were obtained in the axial plane with $2.5-\mathrm{mm}$ or $2-\mathrm{mm}$ thickness and coronal plane with 1-mm thickness. The inner boundary of the orbital bone was traced on each section with the Rapidia Image Post-processing System (Infinitt Co., Ltd., Seoul, Korea) (Fig. 1). Axial sections were used to integrate OV in group $\mathrm{M}$, and coronal sections were used to integrate $\mathrm{OV}$ in groups I and IM. The OV was computed by summing the volumes between the two scan sections, which was calculated by averaging the area of 2 adjacent scan sections and multiplying by section thickness: Volume $=\Sigma\left(\right.$ slice $_{\mathrm{n}}+$ slice $\left._{\mathrm{n}+1}\right) / 2 \times$ thickness [4]. The contralateral OV was used as a control to standardize individual OV differences, and the OVR was obtained by dividing the volume of the traumatized orbit by that of the normal (control) side [9].

Orbital volume ratio $(\%)=\frac{\text { Orbital volume of traumatized side }}{\text { Orbital volume of normal side }} \times 100$

\section{Ophthalmic examination}

Measurements of enophthalmos were performed 6 months after trauma to minimize the influence of periorbital swelling. The enophthalmos was measured with a Hertel exophthalmometer (Inami Inc., Tokyo, Japan) by measuring from the lateral orbital rim to the apex of the cornea on each side [8]. Enophthalmos is defined as the difference between the Hertel values of each side. Posterior displacement of the eye was recorded as a positive value by observers.

Enophthalmos $=$ Hertel value of normal eye-Hertel value of traumatized eye

\section{Fig. 1. CT images used to calculate OVR values}

(A) This patient had $1 \mathrm{~mm}$ of enophthalmos with an OVR of 105.98\% and PFR of $87.15 \%$. (B) This patient had 2 mm of enophthalmos with an OVR of $112.86 \%$ and PFR of $85.91 \%$. (C) This patient had $3 \mathrm{~mm}$ of enophthalmos with an OVR of $115.95 \%$ and PFR of $80.32 \%$. CT, computed tomography; OVR, orbital volume ratio; PFR, palpebral fissure ratio.

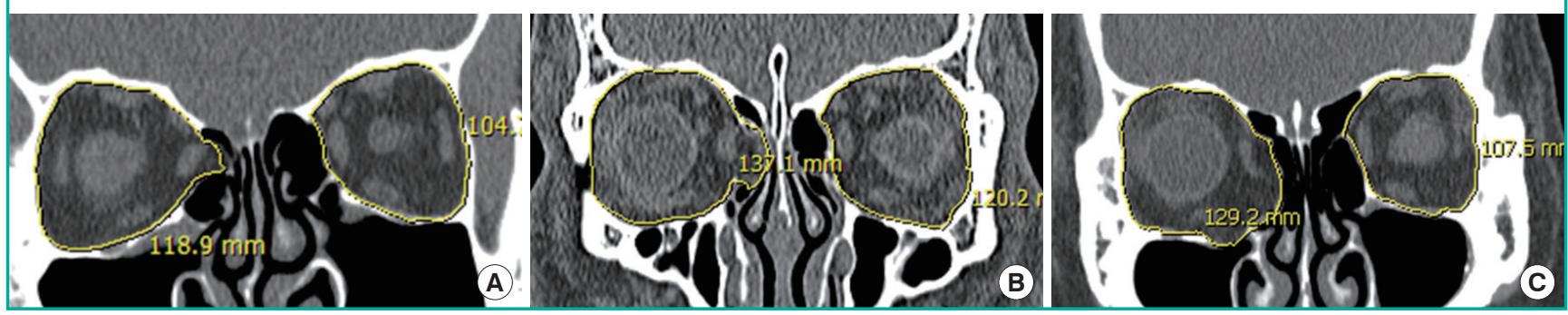




\section{Palpebral fissure measurement}

To evaluate palpebral change, we measured the palpebral fissure ratio (PFR). The PFR was measured by digital photography and computerized image analysis. We took pictures of each patient in the primary gaze position 6 months after trauma to minimize the influence of periorbital swelling. Adobe Photoshop CS5 for Windows (Adobe Systems, San Jose, CA, USA) was used to measure the PFR. After tracing the upper and lower eyelid fissure area of the traumatized side, the number of pixels was calculated using the pen tool and the histogram window in Photoshop. The fissure area of the normal eye was also calculated, and we divided the value for the traumatized side by that of the normal side.

Palpebral fissure ratio $(\%)=\frac{\text { The pixels of traumatized side area }}{\text { The pixels of normal side area }} \times 100$

\section{Statistical analysis}

The relationships between the change in the OVR and both the degree of enophthalmos and the PFR were assessed by using the Pearson product-moment correlation coefficients. A linear regression analysis was performed to obtain the correlation between the extent of enophthalmos and the OVR. A P $<0.05$ was considered significant. All analyses were performed using SPSS ver. 20.0 for Windows (IBM Corp., Armonk, NY, USA).

\section{RESULTS}

The distribution of the 38 patients is shown in Table 1 . The mean age of the patients ( 32 male and 6 female) was $41.81 \pm 11.45$ years (range, $18-58$ years). Twenty-four of the 38 fractures were on the left side, and 14 were on the right side. The mean OVR was $106.84 \% \pm 4.48 \%$, the mean enophthalmos was $1.25 \pm 0.83$ $\mathrm{mm}$, and the mean PFR was $90.72 \% \pm 7.92 \%$ in all patients (Table 2). Enophthalmos increased in proportion to the OVR, and there was a highly significant correlation between the increase in the OVR and the degree of enophthalmos $(\mathrm{P}<0.05)$ (Fig. 1). On the other hand, there was no correlation between OVR and PFR $(\mathrm{P}>0.05)$ (Table 2). According to linear regression analysis, enophthalmos increases by up to $0.145 \mathrm{~mm}$ with every $1 \%$ increase in OVR. This yields the formula $\mathrm{E}=0.145 \mathrm{~V}-14.267$ $(\mathrm{r}=0.77, \mathrm{P}<0.05, \mathrm{n}=38)$ where $\mathrm{E}=$ enophthalmos $(\mathrm{mm})$, $\mathrm{V}=$ OVR (\%). In the above formula, the $\mathrm{r}^{2}$ value was 0.60 , or $60 \%$, which is statistically significant. We calculated the OVR that caused 2-mm enophthalmos in our patient group. Based on the above formula, OVR that induced 2-mm enophthalmos was about $112.18 \%$ in all patients, $112.02 \%$ in group I, $110.40 \%$ in group IM, and $113.12 \%$ in group M (Table 2). Group I showed the steepest gradient relationship between the change in OVR and the degree of enophthalmos among the 3 groups (Fig. 2).

\section{Table 1. Patients' distribution}

\begin{tabular}{|c|c|c|c|c|c|c|c|c|c|c|c|c|c|}
\hline \multirow{2}{*}{$\begin{array}{l}\text { Enophthalmos } \\
(\mathrm{mm})\end{array}$} & \multicolumn{3}{|c|}{$100 \% \leq 0 \mathrm{VR}<105 \%$} & \multicolumn{3}{|c|}{$105 \% \leq 0 \mathrm{VR}<110 \%$} & \multicolumn{3}{|c|}{$110 \% \leq 0 \mathrm{VR}<115 \%$} & \multicolumn{3}{|c|}{$115 \% \leq 0 \mathrm{VR}<120 \%$} & \multirow{2}{*}{ Tota } \\
\hline & I & IM & $M$ & I & IM & $M$ & I & IM & $M$ & I & IM & $M$ & \\
\hline $1.0>E$ & 3 & - & 5 & - & - & - & - & - & - & - & - & - & 8 \\
\hline $1.0 \leq E<1.5$ & - & 2 & 3 & 2 & 2 & 3 & - & - & - & - & - & - & 12 \\
\hline $1.5 \leq E<2.0$ & - & 1 & - & 1 & - & 3 & - & - & - & - & - & - & 5 \\
\hline $2.0 \leq E<3.0$ & - & 1 & - & - & 3 & 3 & - & 2 & - & - & - & 2 & 11 \\
\hline $3.0 \leq E$ & - & - & - & - & - & - & - & - & - & 1 & 1 & - & 2 \\
\hline Total & 3 & 4 & 8 & 3 & 5 & 9 & - & 2 & - & 1 & 1 & 2 & 38 \\
\hline
\end{tabular}

Table 2. Patients' demographics and the correlation analysis between OVR with enophthalmos and PFR

\begin{tabular}{|c|c|c|c|c|c|c|c|c|c|c|}
\hline \multirow[b]{2}{*}{$\begin{array}{l}\text { Fracture } \\
\text { location }\end{array}$} & \multirow[b]{2}{*}{ No. } & \multirow[b]{2}{*}{ Age (yr) } & \multirow[b]{2}{*}{ OVR (\%) } & \multicolumn{4}{|c|}{ Enophthalmos (mm) } & \multicolumn{3}{|c|}{ PFR (\%) } \\
\hline & & & & $\begin{array}{l}\text { Mean } \\
\text { value }\end{array}$ & $\begin{array}{l}\text { Correlation } \\
\text { coefficient }^{\text {a) }}\end{array}$ & P-value ${ }^{a)}$ & $\begin{array}{c}\text { OVR (\%) } \\
\text { induce } \\
\text { enophthalmos } \\
2 \mathrm{~mm}\end{array}$ & $\begin{array}{l}\text { Mean } \\
\text { value }\end{array}$ & $\begin{array}{l}\text { Correlation } \\
\text { coefficient }^{\text {b) }}\end{array}$ & P-value ${ }^{\text {b) }}$ \\
\hline Group I & 7 & 42.71 & 106.40 & 0.92 & 0.977 & $<0.001^{*}$ & 112.02 & 92.70 & -0.749 & 0.053 \\
\hline Group IM & 12 & 40.66 & 107.74 & 1.70 & 0.734 & $0.007^{*}$ & 110.40 & 86.59 & -0.099 & 0.759 \\
\hline Group M & 19 & 42.21 & 106.44 & 1.07 & 0.725 & $<0.001^{*}$ & 113.12 & 92.61 & -0.138 & 0.572 \\
\hline Total & 38 & $41.81 \pm 11.45$ & $106.84 \pm 4.48$ & $1.25 \pm 0.83$ & 0.779 & $<0.001^{*}$ & 112.18 & $90.72 \pm 7.92$ & -0.307 & 0.061 \\
\hline
\end{tabular}




\section{Fig. 2. OVR versus enophthalmos}

Correlations between the OVR and the degree of enophthalmos. There are different slopes in each group. The inferior wall fracture group (group I) showed a steeper slope than the medial wall fracture group (group M). OVR, orbital volume ratio; M, inferomedial wall.

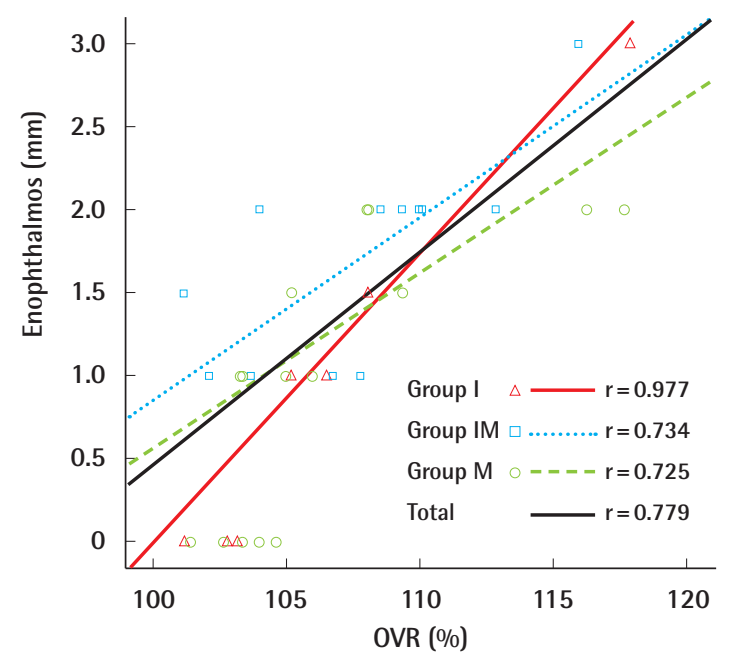

\section{DISCUSSION}

Determination of the operative treatment for blowout fracture patients was based on several factors, including restricted ocular motility, enophthalmos, and the severity of the fractures [1]. However, those operative indications caused some confusion for clinical use and did not yield any objective preoperative numerical guidelines that could estimate eventual enopthalmos degree for determining the need for surgery. When the patients did not have any limitation of ocular movement, the ophthalmic surgeon needed to determine whether to perform the operation by evaluating the preoperative $\mathrm{CT}$ image and the enophthalmos measurements [10]. But, enophthalmos might not appear in the preoperative period due to periorbital swelling, and the preoperative enophthalmos measurements would not be useful as a reliable surgical guideline [11]. Most physicians agree that it is better to perform surgery within 2 weeks after injury, if possible [12]. Therefore, ophthalmic surgeons should be able to predict the eventual final stable degree of enophthalmos with the information from the preoperative CT image and use this to determine whether to perform surgery even if the swelling remains.

Enophthalmos occurs with an increase in volume of the bony orbit with consequent posterior displacement of the globe, but it may not develop immediately after trauma due to orbital swelling $[3,4]$. In fact, its onset can occasionally be delayed for a few weeks, and the final degree of enophthalmos could stabilize a few months after the resolution of periorbital swelling [2]. In other words, the position of the globe in the early post-trauma period may not be representative of the final globe position. There have been many attempts to establish the relationship between OV and enophthalmos in orbital wall fractures [4]. Whitehouse et al. [2] showed that enophthalmos occurred at a depth of $0.8 \mathrm{~mm}$ when the OV increased by $1 \mathrm{~cm}^{3}$. Raskin et al. [5] documented that $0.47 \mathrm{~mm}$ of enophthalmos occurred per 1 $\mathrm{cm}^{3}$ of OV expansion. However, those studies did not consider individual OV difference.

In this study, we tried to demonstrate that the preoperative CT OV measurement could be used as quantitative data to predict final enophthalmos degree in pure blowout fractures and used the OVR-comparing the traumatized eye volume with the normal side - to standardize the individual OV variability. There were significant correlations between enophthalmos and OVR $(\mathrm{P}<0.05)$ (Table 2).

Designating the extent of enophthalmos that is noticeable remains controversial, but many studies have agreed that $2 \mathrm{~mm}$ of relative enophthalmos should be considered cosmetically significant [6-8]. Moreover, most studies have presented an enophthalmos of greater than $2 \mathrm{~mm}$ as the indication for orbital wall surgery [6-8].

In this study, we subdivided the patients into 3 groups depending on the fracture location: group I (inferior wall), group $M$ (medial wall), and group IM (inferomedial wall). We analyzed the correlation between the OVR and enophthalmos according to the fracture location and found significant differences among all 3 groups (Table 2), with steeper slopes in inferior wall fractures than medial wall fractures (Fig. 2). This means that more enophthalmos occurred in the inferior wall fracture group than the medial wall fracture group with the same OVR. It might be that the medial wall was supported by the ethmoid bone, but there was no bony support in floor fractures, and there was an effect from gravity [13]. Thus, we presumed that there might be a greater need for surgical intervention in floor fractures than in those of the medial wall with the same OVR.

Generally, the traumatized enophthalmic eye showed a smaller palpebral fissure size than the normal eye; therefore, we evaluated the PFR to compare eye size, but we could not find a significant correlation with the OVR. This was because palpebral fissure size is affected by many other factors when compared with bony OV, which usually has similar size on either side. Further studies considering other factors are thus needed to search for a significant difference in the PFR.

This study has some limitations. We used preoperative CT of unoperated patients to find OVR that induced 2-mm enophthalmos; therefore, severe operated blowout fracture patients were excluded. In addition, the number of patients with medial wall fractures was greater than that of those with another frac- 
ture location. It was estimated that ocular symptoms that require operation are more commonly associated with floor fracture than medial wall fracture, thus it was difficult to find unoperated floor fracture patients. A larger number of patients and more balanced patient samples among groups are required for more accurate comparison, and further studies should be conducted on the other factors that could influence enophthalmos.

\section{REFERENCES}

1. Rodriguez ED, Dorafshar AH, Manson PN. Facial fractures. In: Neligan PC, Rodriguez ED, Losee JE, editors. Plastic surgery. Philadelphia: Elsevier Saunders; 2013. p.49-88.

2. Whitehouse RW, Batterbury M, Jackson A, et al. Prediction of enophthalmos by computed tomography after 'blow out' orbital fracture. Br J Ophthalmol 1994;78:618-20.

3. Ahn HB, Ryu WY, Yoo KW, et al. Prediction of enophthalmos by computer-based volume measurement of orbital fractures in a Korean population. Ophthal Plast Reconstr Surg 2008;24:36-9.

4. Fan X, Li J, Zhu J, et al. Computer-assisted orbital volume measurement in the surgical correction of late enophthalmos caused by blowout fractures. Ophthal Plast Reconstr Surg 2003;19:207-11.

5. Raskin EM, Millman AL, Lubkin V, et al. Prediction of late enophthalmos by volumetric analysis of orbital fractures. Ophthal Plast Reconstr Surg 1998;14:19-26.
6. Gart MS, Gosain AK. Evidence-based medicine: orbital floor fractures. Plast Reconstr Surg 2014;134:1345-55.

7. Sung YS, Chung CM, Hong IP. The correlation between the degree of enophthalmos and the extent of fracture in medial orbital wall fracture left untreated for over six months: a retrospective analysis of 81 cases at a single institution. Arch Plast Surg 2013;40:335-40.

8. Koo L, Hatton MP, Rubin PA. When is enophthalmos "significant"? Ophthal Plast Reconstr Surg 2006;22:274-7.

9. Oh SA, Aum JH, Kang DH, et al. Change of the orbital volume ratio in pure blow-out fractures depending on fracture location. J Craniofac Surg 2013;24:1083-7.

10. Kwon J, Barrera JE, Jung TY, et al. Measurements of orbital volume change using computed tomography in isolated orbital blowout fractures. Arch Facial Plast Surg 2009;11:3958.

11. Mazock JB, Schow SR, Triplett RG. Evaluation of ocular changes secondary to blowout fractures. J Oral Maxillofac Surg 2004;62:1298-302.

12. Saiepour D, Messo E, Hedlund AJ, et al. Radiologic and longterm clinical outcome from treatment of isolated medial orbital wall blowout fractures. J Craniofac Surg 2012;23:12525.

13. Jo A, Rizen V, Nikolic V, et al. The role of orbital wall morphological properties and their supporting structures in the etiology of "blow-out" fractures. Surg Radiol Anat 1989;11: 241-8. 